\title{
Realidad Aumentada en la educación
}

Bladimir Díaz-Campos ${ }^{1}$

Docente hora-clase Utec

bladimir.diaz@mail.utec.edu.sv

Recibido: 08/03/2016 - Aceptado: 06/04/2016

\section{Resumen}

El uso de las tecnologías en la vida diaria se vuelve cada vez más necesario. La educación sobre todo en los jóvenes, el uso de las tecnologías rivaliza mucho por la atención tanto dentro como fuera del salón de clases compitiendo por el tiempo invertido para la adecuada comprensión y aprendizaje. Una de esas tecnologías que está ganando terreno es la Realidad Aumentada, la cual al contrario de la realidad virtual, define sobre los objetos reales objetos digitales creados por computadora, En la actualidad en el mercado existe una infinidad de soluciones que involucran a la Realidad Aumentada, entre las más conocidas está la de la publicidad, por lo que adentrarse a este mundo y sobre todo para la educación, conlleva a una serie de cuestionamientos y adecuaciones necesarias de la tecnología para esta rama en particular.

\section{Palabras clave}

Realidad Aumentada, educación, Android, dispositivos móviles.

\section{Abstract}

The use of technology in our daily lives, it becomes increasingly necessary. Education and especially for young people, use technology is rivaling much attention both in the classroom and outside of them competing for the invested for a proper understanding and learning time. One of those technologies that is gaining ground is Augmented Reality, which unlike virtual reality, defined on real objects digital objects created by computers, now and in the market there exists a myriad of solutions involving Augmented Reality, among the best known is the advertising, so enter this world and especially for education leads to a series of questions and adaptations of technology for this particular branch.

\section{Keywords}

Augmented reality, education, Android, mobile devices. 


\section{Realidad Aumentada en la educación}

La educación también está empezando a sacar provecho de las aplicaciones móviles (apps) y de la Realidad Aumentada (RA). Tanto para profesores como para alumnos, las apps educativas de RA pueden proporcionar herramientas de aprendizaje muy entretenidas y útiles, explotando el componente visual como su máximo atractivo, utilizando animaciones y vídeos. Por ejemplo, la RA cobra gran importancia en las materias que requieren una dimensión más práctica, como la Física y la Química.

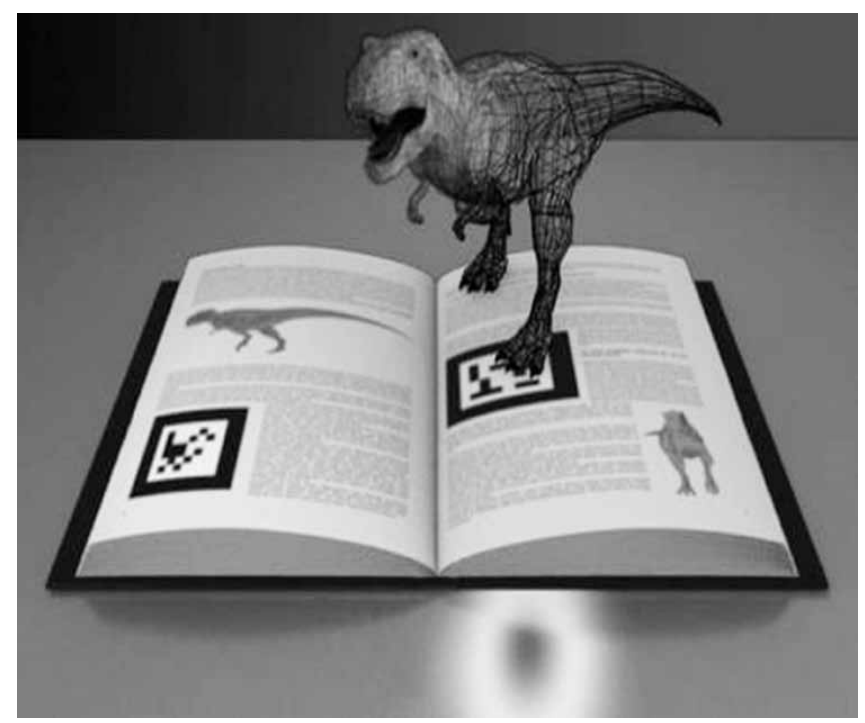

De esta manera, es de vital importancia que en el ámbito educacional sea impulsado por la tecnología y una manera de impacto es desarrollar una aplicación en un entorno de RA en donde la enseñanza para los niños es más importante y atractiva que los juegos de violencia actuales, ya que estos crean perturbación en la enseñanza y no fundamentan ningún avance para su educación y mucho menos para el futuro de nuestro país.

El objetivo principal del proyecto es crear la aplicación Collage Kid's y que esta sea funcional tanto para docentes como para estudiantes. Para lograr la obtención de este objetivo es imprescindible investigar acerca de la tecnología que se empleará y gestionar eficientemente el proyecto en sus diferentes fases.

A nivel de grupo, el objetivo es mediante los conocimientos adquiridos previamente sobre RA, poder ser capaces de gestionar correctamente el proyecto, mejorar los conocimientos en el desarrollo de aplicaciones sobre RA para plataforma Android y toda la tecnología relacionada con el proyecto.

\section{Método}

\section{Diseño y tipo de investigación}

Esta actividad está orientada a utilizar los dispositivos móviles a través de la app Aurasma, para que los niños de 4 a 6 años comiencen a identificar los sonidos y las grafías de las letras del alfabeto, teniendo como objetivos los siguientes:

- Despertar en los niños el interés por el aprendizaje de la lectoescritura

- Vivencia con la articulación de los sonidos de las letras

- Desarrollar la memoria y la creatividad.

- Diferenciar la letra de otras representaciones gráficas.

- Utilizar una buena articulación para los diferentes sonidos.

- Mejorar la percepción y la discriminación auditiva.

- Iniciarse en la utilización de los dispositivos móviles y tabletas con sistema operativo Android para el aprendizaje de la lectoescritura.

Al inicio del proyecto se va presentando tanto la grafía como el sonido y el gesto de las letras, partiendo del nombre del encargado y de la fecha del día.

Se presentan todas las letras, de la $A$ a la $Z$. En un primer momento son letras sueltas, pero poco a poco los niños van representando las letras de su nombre; y al escribirlas todas, una al lado de la otra, ya hay niños que se dan cuenta de que lo que hemos escrito es el nombre de la maestra.

Grabamos a los peques realizando el sonido y gesto de las letras y creamos RA con la página web de Aurasma.

En la esquina de las letras hemos colocado la tableta y los móviles; todas las letras que genera la RA, en papel bond, con el nombre y la foto de la clase. Allí se dirige el maestro del día junto con el responsable del día anterior. 
Al principio solo les interesa ver las letras que ellos han hecho, así que enfocan una y otra vez su letra... Poco a poco va pasando a enfocar diferente letra.

\section{Participantes, universo y muestra}

Los participantes son niños de 4 a 6 años, como personas en las cuales se enfoca primordialmente la aplicación.

\section{Instrumento}

\section{1. ¿Posee un smartphone, una tablet o iPad?}

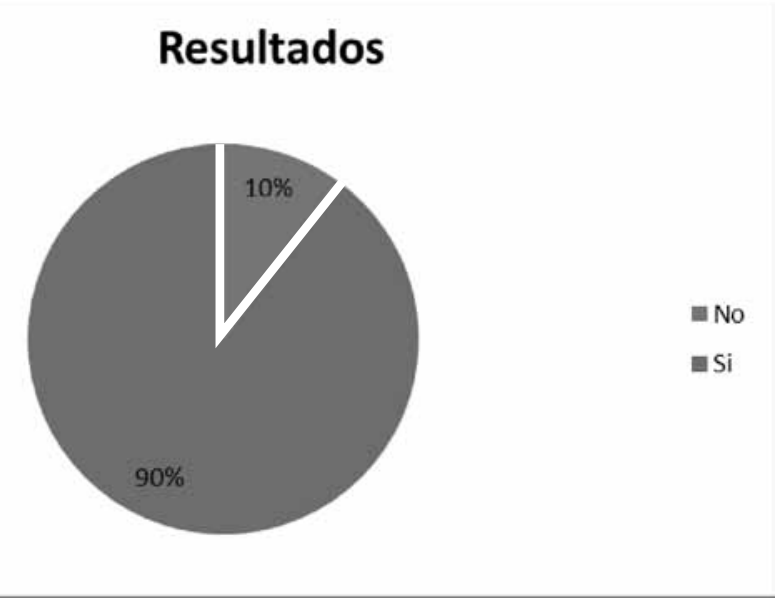

\section{2. ¿Ha escuchado sobre Realidad Aumentada?}

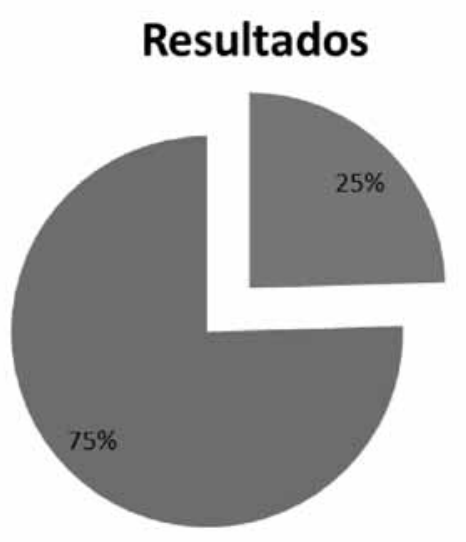

\begin{tabular}{|c|c|}
\hline Frecuencia & Respuesta \\
\hline 13 & No \\
\hline 40 & Sí \\
\hline 1 & NR \\
\hline
\end{tabular}

ANÁLISIS. En los resultados podemos ver en esta pregunta, un $90 \%$ de las personas entrevistadas dijo que sí posee un smartphone, una tablet o un iPad, mientras un $10 \%$ dijo que no. Con esto se concluye que la mayoría de personas poseen uno de estos.

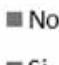

ANÁLISIS. En los resultados podemos ver en esta pregunta, un $75 \%$ de las personas entrevistadas dijo que no conocen de la tecnología; un $25 \%$ dijo no saber nada de ella. 


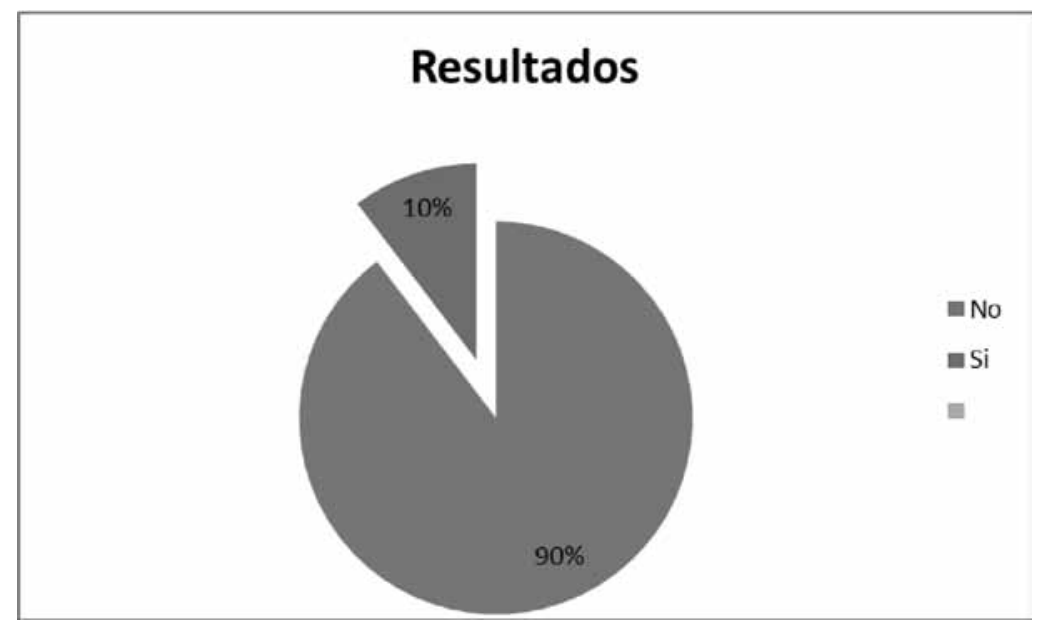

4. ¿Puede utilizar adecuadamente las aplicaciones Android?
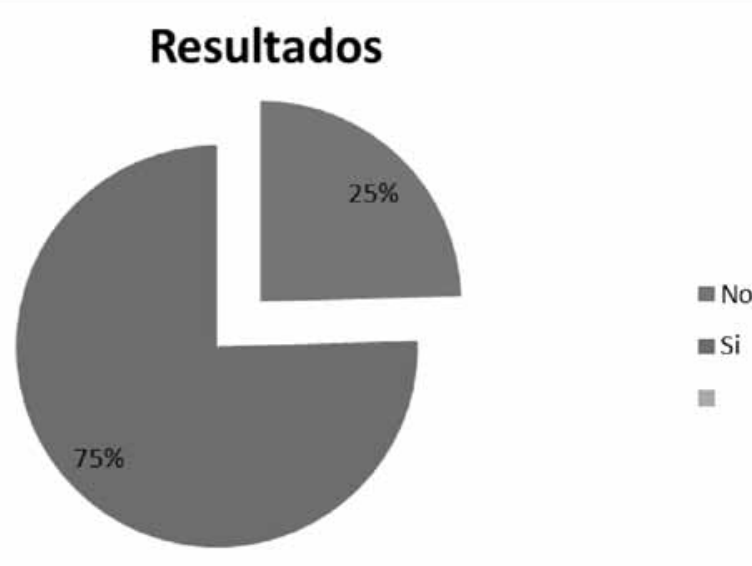

\begin{tabular}{|c|c|}
\hline Frecuencia & Respuesta \\
\hline 14 & No \\
\hline 43 & Sí \\
\hline 1 & NR \\
\hline
\end{tabular}

ANÁLISIS. En los resultados podemos ver en esta pregunta, un $90 \%$ de las personas entrevistadas dijo que no conoce ninguna aplicación sobre Realidad Aumentada y un $10 \%$

dijo no saber nada de ella.

\begin{tabular}{|c|c|}
\hline Frecuencia & Respuesta \\
\hline 52 & No \\
\hline 6 & Sí \\
\hline
\end{tabular}

ANÁLISIS. En esta pregunta un $75 \%$ de la muestra dijo que sí sabe utilizar las app Android, mientras un $25 \%$ dijo que no. Con esto confirmamos que la mayoría puede usar adecuadamente las app Android. 


\section{5. ¿Le sería útil probar una aplicación que le muestre el aprendizaje de lenguaje?}

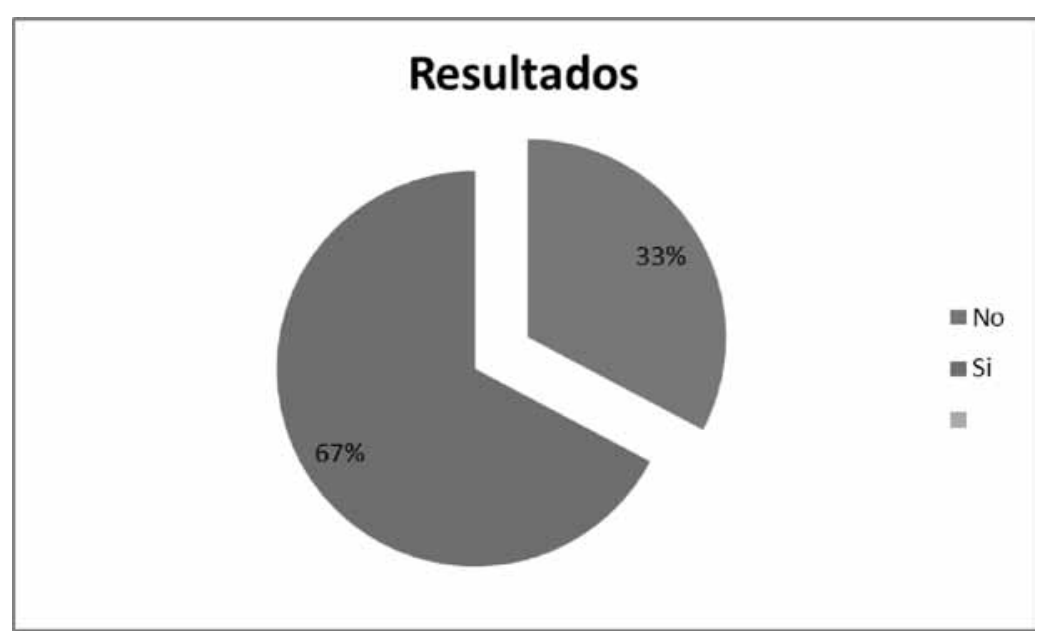

6. ¿Cómo valorarías la aplicación?

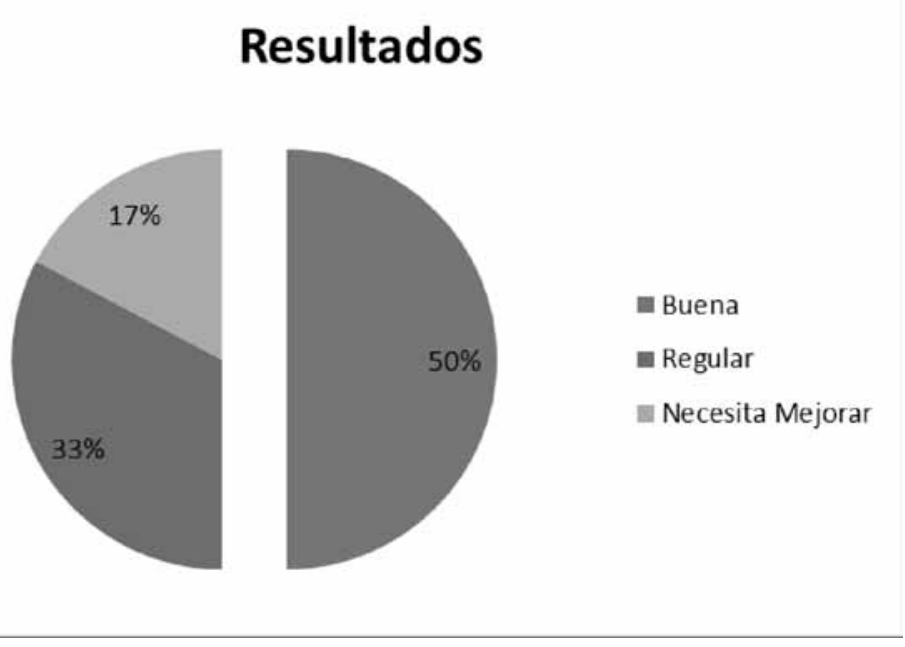

\begin{tabular}{|c|c|}
\hline Frecuencia & Respuesta \\
\hline 19 & No \\
\hline 39 & Sí \\
\hline
\end{tabular}

ANÁLISIS. En los resultados podemos observar en esta pregunta, un $67 \%$ de la muestra dijo que sí sería útil probar dicha app, mientras un $33 \%$ dijo que no. Con esto tenemos que la mayoría de los entrevistados eran estudiantes.

\begin{tabular}{|c|c|}
\hline Frecuencia & Respuesta \\
\hline 29 & Buena \\
\hline 19 & Regular \\
\hline 10 & $\begin{array}{c}\text { Necesita } \\
\text { Mejorar }\end{array}$ \\
\hline
\end{tabular}

ANÁLISIS. En esta pregunta un $50 \%$ dijo que valoraría dicha app como buena; un $33 \%$ dijo que la valoraría como regular; mientras que un $17 \%$ dijo que debe de mejorar. En conclusión, la mayoría de entrevistados valora en un rango de buena dicha app. 


\section{7. ¿Recomendarías esta aplicación a tus amigos?}

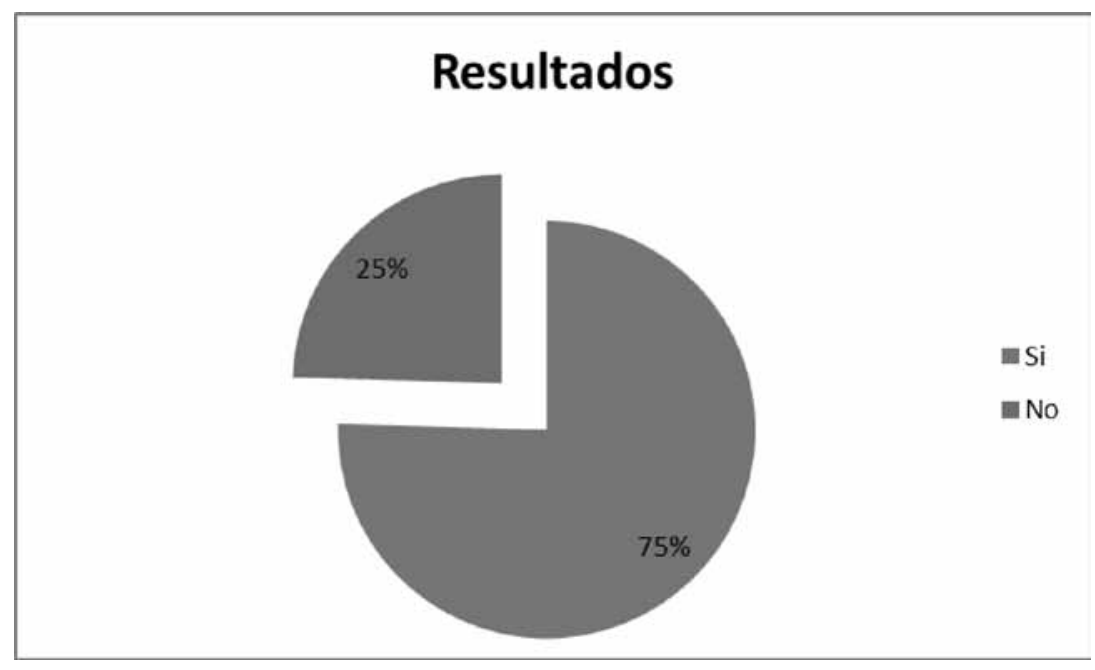

\begin{tabular}{|c|c|}
\hline Frecuencia & Respuesta \\
\hline 43 & Sí \\
\hline 14 & No \\
\hline 1 & NR \\
\hline
\end{tabular}

ANÁLISIS. Como respuesta a la última pregunta, los datos obtenidos dicen que un $75 \%$ recomendaría esta app, mientras que un $25 \%$ dijo que no. Con esto se concluye que la app sería recomendada por la muestra.

\section{Resultados}

Con esta app se espera que muchas escuelas e instituciones la puedan tomar como un material didáctico muy útil, según sea la enseñanza.

La aplicabilidad en la cual se basa más en la app es su uso cotidiano, en el cual no solo puede ser tomada como una herramienta, sino como un material didáctico que se utilicé ampliamente como una motivación para desarrollar herramientas de enseñanza académicas y de una amplia compresión de cualquier tema que se trate, de la siguiente forma:

- Estrictamente los resultados, evidenciando cada aspecto.

- Pueden colocarse tablas o figuras, así como análisis.

También en este apartado se pueden incluir tablas o figuras, toda vez que reflejen los resultados.

Tabla 1

Datos Sociodemográfico

\begin{tabular}{|c|c|c|c|c|c|c|c|}
\hline & $M(S D)$ & A & B & C & D & $E$ & $F$ \\
\hline Color & $1.53(.50)$ & & .07 & -.09 & .02 & .14 & .06 \\
\hline Género & $\begin{array}{c}31.88 \\
(10.29)\end{array}$ & & & .08 & $.19^{*}$ & $.20^{*}$ & .01 \\
\hline Ingresos & $2.60(1.57)$ & & & & .04 & -.14 & -.09 \\
\hline Nivel Esc. & $3.44(1.06)$ & & & & & $-.29^{*}$ & -.06 \\
\hline Religión & $1.21(.30)$ & & & & & & $-.19^{*}$ \\
\hline Dist. Intol. & $3.75(1.19)$ & & & & & & \\
\hline
\end{tabular}




\section{Discusión/conclusión}

Conforme se va desarrollando la globalización y se generan nuevas tecnologías, es necesario tener la información en tiempo oportuno; y una de estas tecnologías en auge son los dispositivos móviles. Con ellos se puede tener acceso a internet y a las diversas aplicaciones que proporcionan sus sistemas operativos.

Nuestra app ayudará a los estudiantes a tener más y mejor apoyo en su aprendizaje y para la memorización de diferentes temas educativos, lo que será muy beneficioso para los alumnos, para que ya no tengan el paradigma de que los celulares y las aplicaciones son, más que todo, para el ocio, aplicando tecnologías recientes y de mayor visualización, para que el estudiante tenga una mayor interacción para el aprendizaje.

El software educativo servirá como apoyo a las actividades de un estudiante en la escuela o instituto, que evidenciará un cambio favorable en el sistema educativo del país, pues es una alternativa válida que ofrece al usuario un ambiente propicio para la construcción del conocimiento.

El diseño de la aplicación educativa facilitará el aprendizaje sobre la identificación de fórmulas previa a una actividad.
En este trabajo la informática debe ser aplicada obligatoriamente en un contexto educativo; el desconocimiento de las teorías que le dan sentido en el más claro ejemplo de ignorancia en cuanto a falta de cultura informática en la actualidad.

\section{Referencias}

Hewlett Packard Development Company (2016). "Get Started". Recuperado de https://www.aurasma.com/

Mocholí, A. (2014). Yeeply. "Claves y herramientas para desarrollar aplicaciones móviles de Realidad Aumentada". Recuperado de https://www.yeeply. com/blog/desarrollar-aplicaciones-moviles-derealidad-aumentada/

Molinero, R. ( 2014). "Hablando en las nubes". Recuperado de http://hablandoenlasnubes. blogspot.com.es/2014/06/6-apps-para-empezarusar-la-realidad.html

Navarro, C. ( 2013). "Palabras azules. Las letras con Realidad Aumentada". Recuperado de http://elmarescolorazul. blogspot.com/2013/11/las-letras-con-realidadaumentada.html

Definición ABC. Diccionario en línea. Recuperado de http:// www.definicionabc.com/comunicacion 